\title{
Immunopathological properties of the Campylobacter jejuni flagellins and the adhesin CadF as assessed in a clinical murine infection model
}

Anna-Maria Schmidt ${ }^{1}$, Ulrike Escher ${ }^{1}$, Soraya Mousavi ${ }^{1}$, Nicole Tegtmeyer ${ }^{2}$, Manja Boehm² ${ }^{2}$ Steffen Backert ${ }^{2}$, Stefan Bereswill ${ }^{1}$ and Markus M. Heimesaat ${ }^{1 *}$

\begin{abstract}
Background: Campylobacter jejuni infections constitute serious threats to human health with increasing prevalences worldwide. Our knowledge regarding the molecular mechanisms underlying host-pathogen interactions is still limited. Our group has established a clinical C. jejuni infection model based on abiotic IL-10 $0^{-1}$ mice mimicking key features of human campylobacteriosis. In order to further validate this model for unraveling pathogen-host interactions mounting in acute disease, we here surveyed the immunopathological features of the important C. jejuni virulence factors FlaA and FlaB and the major adhesin CadF (Campylobacter adhesin to fibronectin), which play a role in bacterial motility, protein secretion and adhesion, respectively.

Methods and results: Therefore, abiotic IL-10 ${ }^{-1-}$ mice were perorally infected with C. jejuni strain 81-176 (WT) or with its isogenic flaA $B$ ( $\triangle f l a A / B)$ or $c a d F(\triangle c a d F)$ deletion mutants. Cultural analyses revealed that WT and $\triangle c a d F$ but not $\triangle f l a A / B$ bacteria stably colonized the stomach, duodenum and ileum, whereas all three strains were present in the colon at comparably high loads on day 6 post-infection. Remarkably, despite high colonic colonization densities, murine infection with the $\triangle f l a A / B$ strain did not result in overt campylobacteriosis, whereas mice infected with $\triangle$ cadF or WT were suffering from acute enterocolitis at day 6 post-infection. These symptoms coincided with pronounced pro-inflammatory immune responses, not only in the intestinal tract, but also in other organs such as the liver and kidneys and were accompanied with systemic inflammatory responses as indicated by increased serum MCP-1 concentrations following $C$. jejuni $\triangle c a d F$ or WT, but not $\triangle$ flaA/B strain infection.

Conclusion: For the first time, our observations revealed that the C. jejuni flagellins $A / B$, but not adhesion mediated by CadF, are essential for inducing murine campylobacteriosis. Furthermore, the secondary abiotic $\mathrm{IL}-10^{-/-}$infection model has been proven suitable not only for detailed investigations of immunological aspects of campylobacteriosis, but also for differential analyses of the roles of distinct C. jejuni virulence factors in induction and progression of disease.
\end{abstract}

\footnotetext{
*Correspondence: markus.heimesaat@charite.de

1 Department of Microbiology, Institute of Microbiology, Infectious Diseases and Immunology, Charité-University Medicine Berlin, corporate member of Freie Universität Berlin, Humboldt-Universität zu Berlin, and Berlin Institute of Health, CC5, Campus Benjamin Franklin, FEM, Garystr. 5, 14195 Berlin, Germany

Full list of author information is available at the end of the article
} (http://creativecommons.org/licenses/by/4.0/), which permits unrestricted use, distribution, and reproduction in any medium, provided you give appropriate credit to the original author(s) and the source, provide a link to the Creative Commons license, and indicate if changes were made. The Creative Commons Public Domain Dedication waiver (http://creativecommons.org/ publicdomain/zero/1.0/) applies to the data made available in this article, unless otherwise stated. 
Keywords: Campylobacter jejuni, FlaA/B, CadF, Flagellin, IL-10 $10^{-/-}$mice, Secondary abiotic (gnotobiotic) mice, Proinflammatory immune responses, Host-pathogen-interaction, Bacterial translocation, Intestinal immunopathology, Extra-intestinal immune responses, Systemic immune responses

\section{Background}

Campylobacter jejuni are spiral-shaped, highly motile, Gram-negative bacteria that frequenly asymptomatically colonize birds, including poultry. In humans the bacteria cause campylobacteriosis, the most prevalent cause for enteric bacterial infections [1-4]. Human C. jejuni infections are predominantly caused by consumption of contaminated animal products and surface water [5]. Campylobacteriosis is accompanied with clinical manifestations such as abdominal pain, fever, and watery or bloody diarrhea that are mostly selflimiting $[1,6,7]$. In a minority of cases, severe postinfectious sequelae such as Guillain-Barré syndrome or reactive arthritis can occur $[7,8]$.

The exact molecular mechanisms underlying the development of acute and invasive enterocolitis that is typical for campylobacteriosis are unclear, but the immunopathological nature of the disease has been recognized for decades [6]. We and others have shown that $C$. jejuni interact with pattern recognition receptors such as Toll-like receptor 4 (TLR-4) [9] and nucleotide-oligomerization-domain-2 (Nod2) $[10,11]$, and interfere with signaling pathways dependent on MAPK/ERK (mitogen-activated protein kinases/extracellular signal-regulated kinases) and NF- $\mathrm{BB}$ (nuclear factor kappa-light-chain-enhancer of activated B cells) [12]. Activation of those signaling cascades induces the expression of a variety of immune response genes [13, 14]. As a result, an inflammation response is triggered, characterized by the recruitment of immune cells to the site of infection and up-regulation of cytokine production [14].

As a prerequisite for induced immunopathology, $C$. jejuni needs to adhere to and invade into epithelial host cells. Amongst a number of other factors, the flagellar filaments consisting of FlaA and FlaB, and the major adhesin CadF (Campylobacter adhesin to fibronectin) are considered to be major players in these processes [15]. To adhere to intestinal host cells, the bacteria need to cross the overlying mucus layer by flagella-generated motility [16]. Moreover, the flagellum can secrete molecules that promote $C$. jejuni adhesion to and invasion into host cells [17-20]. The adhesin CadF permits host cell adhesion by binding to the extracellular matrix protein fibronectin, which enables the interaction with integrin receptors and results in bacterial internalization into host cells $[19,21,22]$.
The dependence of adherence and invasion on flagella has been demonstrated in vitro and in vivo by gene knockout experiments [23, 24]. It was also shown that knockout of $c a d F$ resulted in reduced adhesion and invasion of $C$. jejuni into host cells in vitro [21,25] and abolished colonisation in the chicken host [26]. Both the flagellum and CadF also activate a signaling cascade in cultured INT-407 cells and other cell lines that results in the activation of the small Rho GTPase Rac1, which in turn leads to actin and/or microtubule rearrangements that trigger internalization of C. jejuni [27].

In order to study pathogenesis, treatment and prophylaxis of campylobacteriosis in vertebrate hosts in more detail, we have established a murine $C$. jejuni infection model based on secondary abiotic IL-10 $0^{-1-}$ mice that not only allows for investigation of colonisation properties, but also reproducibly displays clinical symptoms resembling those of the compromized infected human host [28-30]. Applying this clinical infection model we have recently shown, for instance, that $C$. jejuni lipooligosaccharide (LOS) is essential for the induction of campylobacteriosis and this pathogen surface molecule thus represent an important $C$. jejuni pathogenicity factor $[28$, $31,32]$.

To further validate this murine infection model for the study of $C$. jejuni virulence factors for induction and progression of acute disease, we here addressed whether bacterial flagella and the major adhesin CadF are pivotal prerequisites for inducing enteric disease in the murine host. To this aim, we infected secondary abiotic IL-10 ${ }^{-/-}$ mice with $C$. jejuni strain 81-176, its isogenic non-motile mutant $\triangle f l a A / B$ and its CadF-deficient $\Delta c a d F$ mutant. The colonization capacities of these isogenic strains were compared, while clinical outcome as well as intestinal, extraintestinal and systemic immunopathologocal responses were monitored and bacterial translocation to extra-intestinal organs was determined.

\section{Results}

The impact of $C$. jejuni motility and adhesion to intestinal colonization following peroral infection of secondary abiotic IL-10 $0^{-/-}$mice

We first determined whether inactivation of $f l a A / B$ or $c a d F$ had an impact on gastrointestinal colonization of C. jejuni in the secondary abiotic IL- $10^{-/-}$mice model, by comparing these mutants with isogenic WT bacteria. Approximately $10^{9}$ viable bacteria of each strain were 
orally fed on days 0 and 1 . As early as $24 \mathrm{~h}$ following the first infection and until the end of the observation period (i.e., day 6 p.i.), median fecal pathogenic loads of up to $10^{9}$ colony-forming units per $\mathrm{g}(\mathrm{CFU} / \mathrm{g})$ were determined for all three tested bacterial strains (Fig. 1). At day 6 p.i., the animals were sacrificed and $C$. jejuni content of the complete gastrointestinal tract was quantified by culture. As expected, the highest loads were present in the ileum and colon, while stomach and duodenum contained approximately four log lower counts (Fig. 2). The numbers of the $\triangle f l a A / B$ mutant were lower in luminal samples taken from the stomach, duodenum, and ileum, compared to the other two strains $(\mathrm{p}<0.001$; Fig. $2 \mathrm{a}-\mathrm{c})$, but no difference was found in the colon (Fig. 2d). Hence, inactivation of $f l a A / B$, but not of $c a d F$, leads to a compromised colonization potential of $C$. jejuni in the proximal gastrointestinal tract, while colonization of the distal part of the gastrointestinal tract is not affected by these gene deficiencies.

Clinical impact of $C$. jejuni motility and adhesion in infected secondary abiotic IL-10 $0^{-/-}$mice

We next addressed whether comparable colonic loads of the respective $C$. jejuni strains were associated with similar pathogen-induced disease outcomes. Whereas mice displayed increasing clinical scores starting at day 2 following infection with WT and $\Delta c a d F$ bacteria, indicative for progressive C. jejuni-induced disease (Fig. 3a, c), infection with the $\triangle f l a A / B$ mutant left the mice clinically uncompromised (Fig. 3b). In fact, by day 6 p.i., mice colonized with the WT strain were suffering from severe signs of campylobacteriosis including wasting and bloody diarrhea, while none of the animals infected with $\Delta f l a A / B$ exerted symptoms, similar to mock infected control mice ( $<<0.001$; Fig. 4a); the $\Delta c a d F$ infected mice, however, displayed slightly lower clinical scores as compared to WT strain infected counterparts $(p<0.005$; Fig. $4 a)$. Hence, despite high intestinal pathogenic loads, murine infection with the $\triangle f l a A / B$ mutant did not result in overt campylobacteriosis, whereas inactivation of the $c a d F$ gene only marginally impaired the ability to cause symptoms.

\section{Relevance of $C$. jejuni motility and adhesion in induction of intestinal apoptosis and epithelial regeneration}

Given that intestinal inflammation is accompanied by shortening of the affected intestinal compartment [28, 33 ], we measured the colonic lengths upon necropsy. Irrespective of the applied strain, $C$. jejuni infected mice exhibited shorter large intestines as compared to mock control animals $(\mathrm{p}<0.001$; Fig. $4 \mathrm{~b})$. The effect was weaker for animals infected with the $\triangle f l a A / B$ mutant, whose colonic lengths were longer compared to parental WT

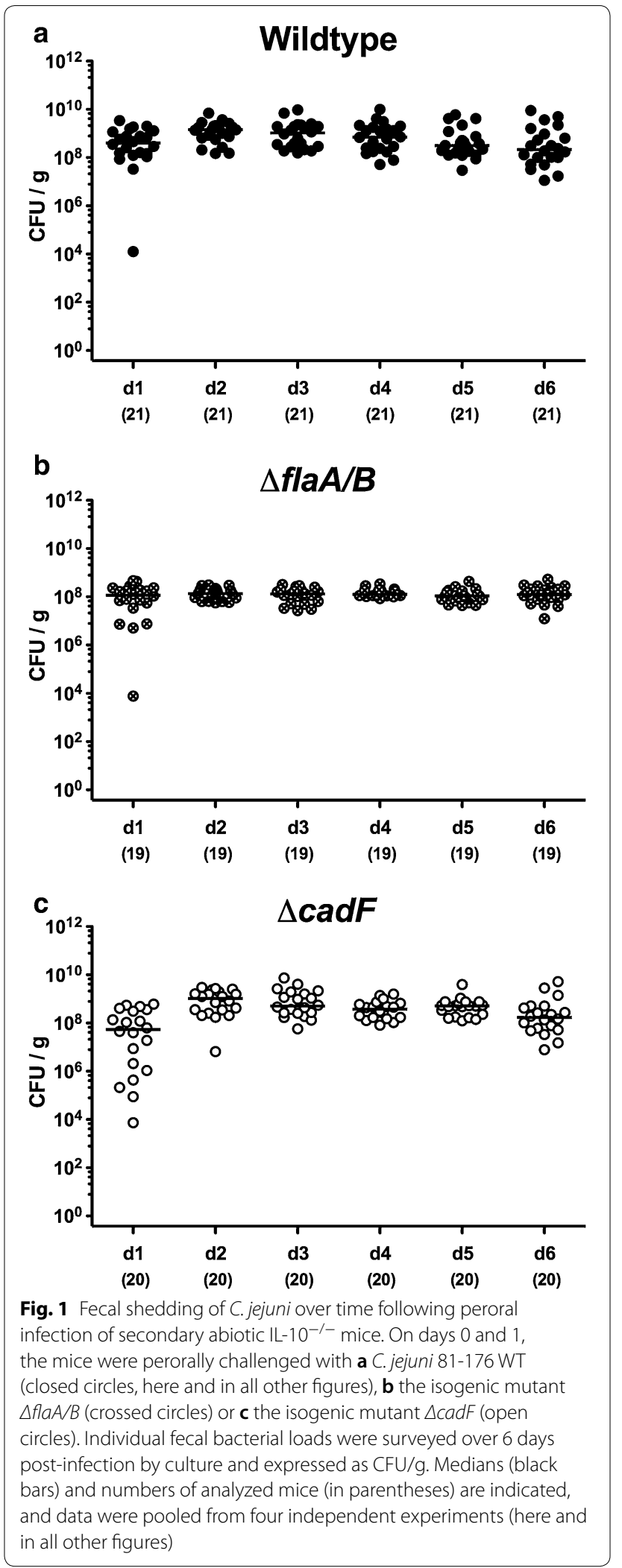




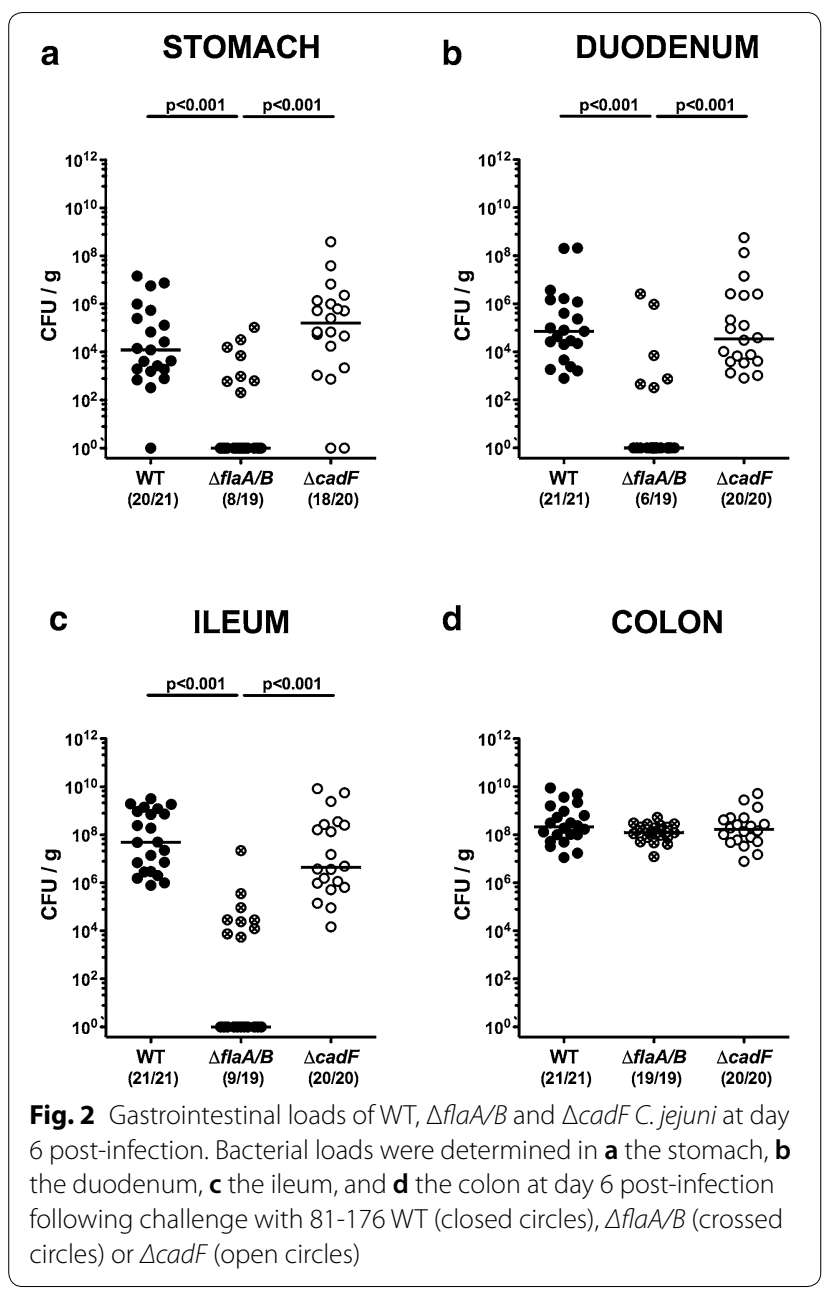

or $\Delta c a d F$ challenge $(\mathrm{p}<0.001$ and $\mathrm{p}<0.01$, respectively; Fig. $4 b)$.

We further addressed whether the lack of apparent symptoms upon $\triangle f l a A / B$ infection could be corroborated microscopically. Given that apoptosis is regarded as a reliable marker for the grading of intestinal inflammatory conditions [31], we quantitatively assessed caspase $3+$ colonic epithelial cell responses. The colonic samples from mice infected with $\Delta c a d F$ and WT contained significantly increased numbers of apoptotic cells ( $p<0.001$ vs naive), whereas slightly lower cells were apoptotic in the colonic epithelia following $\Delta c a d F$ infection compared to WT $(\mathrm{p}<0.001$; Fig. 4c; Additional file 1: Fig. S1A); notably, no increase was observed following $\triangle f l a A / B$ infection. Furthermore, the numbers of Ki67 + cells, indicative for cell proliferation and regeneration, had increased considerably in the colonic epithelia of mice infected with $\Delta c a d F$ or WT bacteria ( $<<0.001$ vs naive), whereas these cell numbers did not differ between $\triangle f l a A / B$ infected and mock infected control mice (Fig. 4d; Additional file 1: Fig. S1B). Hence, in contrast to peroral challenge with WT and the $\triangle c a d F$ mutant, infection with non-motile $C$. jejuni $\triangle f l a A / B$ did neither result in significant macroscopic nor microscopic inflammatory sequelae. These observations make it unlikely that presence of the bacteria in the stomach and duodenum were solely due to coprophagy.

\section{C. jejuni motility and adhesion in induction of colonic immune cell responses}

The three $C$. jejuni strains were also compared for their ability to elicit innate and adaptive immune cell responses within the large intestines of infected mice. Peroral infection with the WT and $\Delta c a d F$, but not the $\triangle f l a A / B$ strain was associated with a marked increase in innate immune cell subsets, such as $\mathrm{F} 4 / 80+$ macrophages and monocytes in the colonic mucosa and lamina propria $(\mathrm{p}<0.001$; Fig. 5a; Additional file 1: Fig. S1C). Adaptive immune cells such as CD3 + T lymphocytes and B220 + B lymphocytes had all increased in the large intestinal mucosa and lamina propria in the case of WT and $\Delta c a d F(\mathrm{p}<0.001)$, but this was not observed during $\triangle$ flaA/B infection (Fig. $5 \mathrm{~b}$, c; Additional file 1: Fig. S1D, E). Interestingly, colonic T cell numbers were even slightly higher in animals that had received $\Delta c a d F$ compared to WT $(\mathrm{p}<0.05$; Fig. $5 \mathrm{~b}$, c; Additional file 1: Fig. S1D). Hence, murine infection with the $C$. jejuni $\Delta c a d F$ mutant and its parental strain, but not with the $\triangle f l a A / B$ mutant, resulted in pronounced innate and adaptive immune cell responses in the large intestines.

\section{C. jejuni motility and adhesion in intestinal pro-inflammatory mediator secretion}

We next measured pro-inflammatory mediators in distinct parts of the intestinal tract following C. jejuni infection. Colonic secretion of TNF- $\alpha$ and nitric oxide was increased exclusively upon infection with WT and $\Delta c a d F$ strains $(\mathrm{p}<0.001$; Fig. 6a, c). Following WT strain infection only, colonic levels of IL- 6 and IFN- $\gamma$ were elevated ( $p<0.01$ and $p<0.001$, respectively; Fig. $6 \mathrm{~b}, \mathrm{~d}$ ), which also held true for nitric oxide and IFN- $\gamma$ concentrations in ex vivo biopsies derived from mesenteric lymph nodes $(\mathrm{MLN})$ at day 6 p.i. $(\mathrm{p}<0.001$ and $\mathrm{p}<0.01$, respectively; Fig. 6e, f).

Hence, murine infection with $\Delta c a d F$, but not $\Delta f l a A / B$ deficient bacteria resulted in enhanced pro-inflammatory mediator secretion in the intestinal tract.

\section{C. jejuni motility and adhesion in extra-intestinal and systemic pro-inflammatory immune responses}

We next assessed whether the immunopathological differences observed between the $C$. jejuni $c a d F$ and $f l a A / B$ mutants were extended to extra-intestinal and even systemic compartments. For extra-intestinal sites, 


\section{a Clinical Conditions - WT}

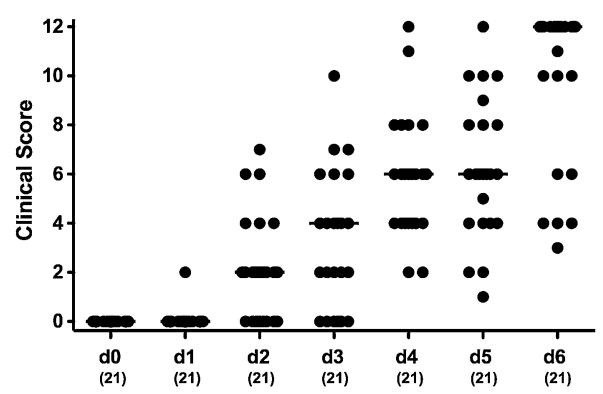

\section{b Clinical Conditions - $\Delta$ flaA/B}

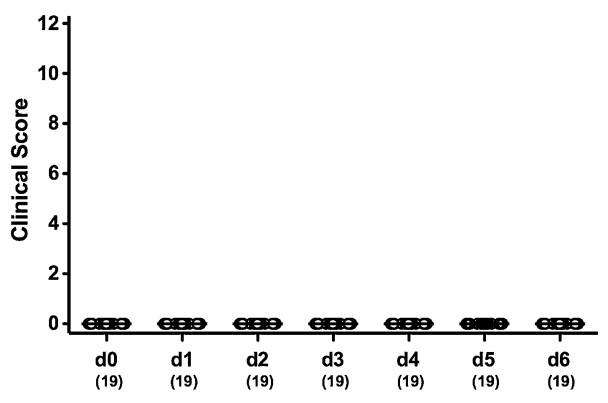

\section{c Clinical Conditions - $\Delta$ cadF}

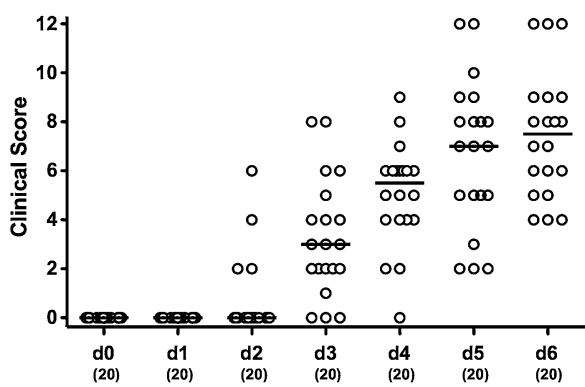

Fig. 3 Clinical conditions over time of mice challenged with the three $C$. jejuni strains. Clinical conditions were monitored during the 6 days post challenge with a WT, $\mathbf{b} \triangle \mathrm{FlaA} B$, and $\mathbf{c} \triangle \mathrm{cadF}$, and these were quantitatively assessed applying a standardized clinical scoring system

we determined numbers of CD3 $+\mathrm{T}$ cells and TNF- $\alpha$ secretion in liver and kidneys. Infection with WT or $\triangle c a d F$ but not $\triangle f l a A / B$ resulted in elevated numbers of $\mathrm{T}$ lymphocytes in either organ $(\mathrm{p}<0.001$; Fig. $7 \mathrm{a}, \mathrm{b})$, and in slightly increased TNF- $\alpha$ secretion in the kidneys ( $\mathrm{p}<0.01-0.001$; Fig. $7 \mathrm{~d}$ ). In the liver, however, only WT strain infection was associated with elevated TNF- $\alpha$ concentrations ( $\mathrm{p}<0.01$; Fig. 8c). Furthermore, straindependent differences in immunopathological responses upon $C$. jejuni infection could also be observed systemically: Mice infected with the WT, but not the $\triangle f l a A / B$ strain produced increased systemic levels of TNF- $\alpha$, IL-6, IFN- $\gamma$, and MCP-1 ( $<<0.001$; Fig. 8), whereas in $\Delta c a d F$ infected mice, elevated MCP-1 serum concentrations could be measured ( $\mathrm{p}<0.05$ vs mock; Fig. $8 \mathrm{~d}$ ).

We finally addressed whether the observed differences in extra-intestinal and systemic pro-inflammatory responses could be due to different levels of translocated bacteria. Samples of various organs were cultured for presence of $C$. jejuni, which revealed their presence in MLN, liver, lungs, and spleen in a number of animals, though all cardiac blood cultures were negative (Fig. 9). The relative abundance of viable bacteria was lower in MLN, liver, lungs, and spleen of animals infected with $\triangle f l a A / B$ compared to the other two strains, while culture of kidney homogenates resulted in fewer positive samples for the two mutants compared to WT. These data indicate that murine infection with $C$. jejuni WT or the $c a d F$ mutant was accompanied with marked extra-intestinal and even systemic pro-inflammatory immune responses, that were absent in case of $\triangle f a A / B$, and that these were paralleled by detectable amounts of viable organisms in various tissue sites. The lower numbers of $\triangle \mathrm{fla} A / B$ bacteria in extra-intestinal organs suggests that these nonmotile bacteria were less able to translocate from the gut to other tissues.

\section{Discussion}

Both the bacterial flagella and the adhesin CadF are well-investigated pathogenicity and virulence factors of C. jejuni, respectively, and are considered key players for colonization and subsequent host cell invasion [2-4]. In vitro studies revealed that CadF-mediated invasion of intestinal epithelial cells represents a crucial prerequisite for $C$. jejuni to initiate immunopathological responses via induction of cytokine responses [19, 21, 22, 34-36]. In fact, these immunopathological sequelae of infection help to explain the severity of symptoms during acute campylobacteriosis which are induced by cells of the innate immune system [37]. In our present study, we provide in vivo evidence that FlaA/B and CadF exert differential features in the interaction of $C$. jejuni and the mammalian host. By means of our clinical murine infection model, we show that $C$. jejuni flagellar motility but not adhesion exerted by CadF is required for induced immunopathology in the murine host. Neither inactivation of the flagellin genes nor of the $c a d F$ gene resulted in a compromized large intestinal colonization by $C$. jejuni as indicated by comparably high colonic loads of either bacterial strain. However, whereas WT bacteria and the 


\section{a}

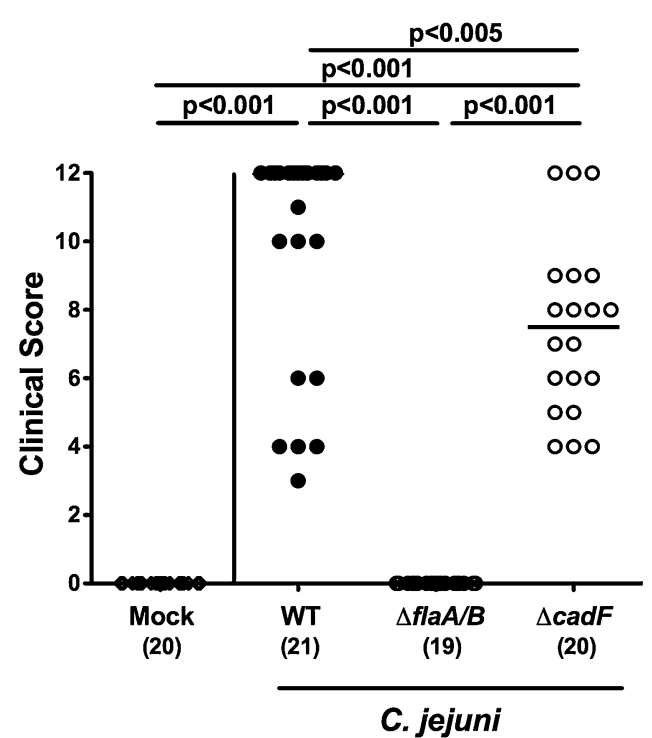

c Apoptotic Cells - COLON

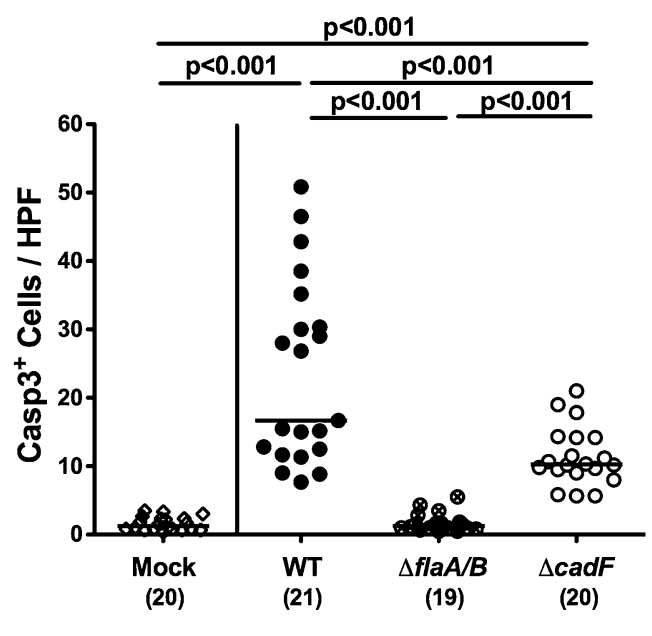

C. jejuni b Colonic Length

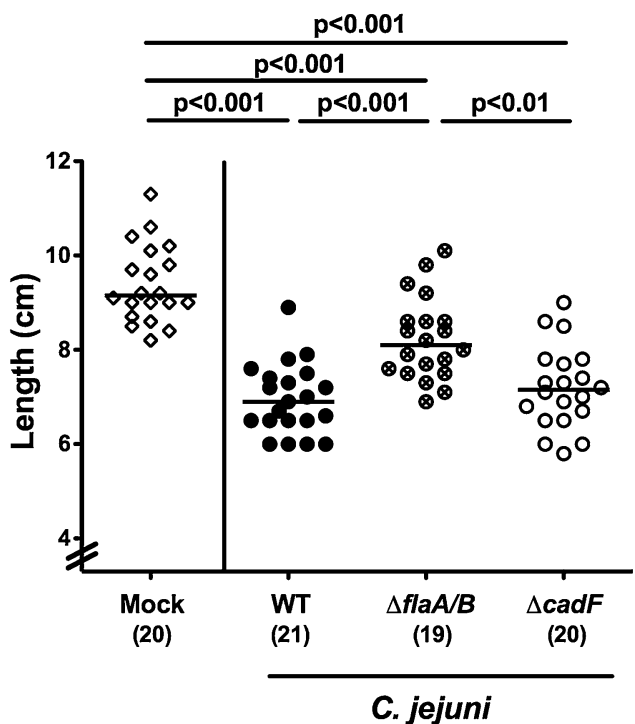

d Proliferating Cells - COLON

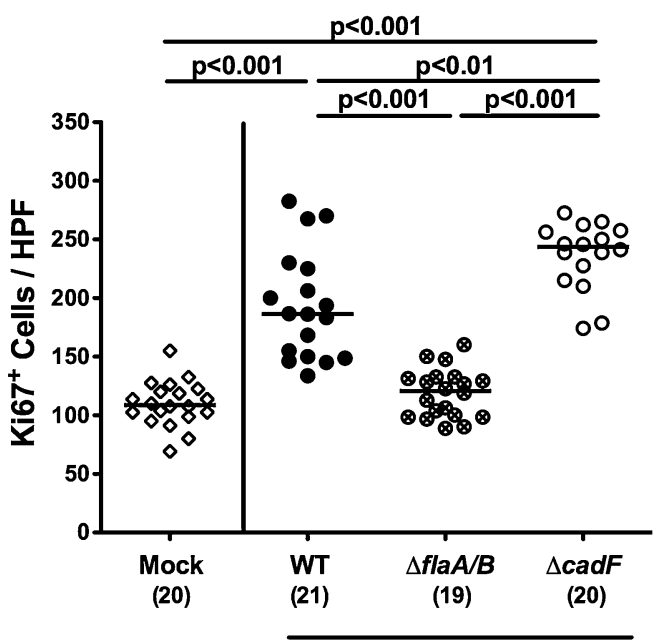

C. jejuni

Fig. 4 Macroscopic and microscopic parameters at day 6 post-infection. Macroscopic C. jejuni induced sequalae determined at day 6 included a clinical conditions and $\mathbf{b}$ colonic length. Microscopic intestinal changes were quantitated by the average numbers of $\mathbf{c}$ colonic epithelial apoptotic cells (positive for caspase-3, Casp3), and $\mathbf{d}$ of proliferating/regenerating cells (positive for Ki67) from six high power fields (HPF, $\times 400$ magnification) per animal in immunohistochemically stained colonic paraffin sections at day 6 post-infection. Mock challenged mice (open diamonds) served as negative controls

cadF deficient mutant could also be isolated from the stomach, duodenum and ileum upon peroral infection, these sites were only poorly colonized by the non-motile mutant strain. This pronounced phenotype provides strong evidence that motility is required to allow $C$. jejun $i$ to escape the unfavorable luminal conditions exerted by acids, bicarbonate and lytic enzymes within the lumen of the upper environmental tract, for instance. In this scenario motility allows the bacteria to reach mucus sites where the pathogen is protected from toxic influences and can adhere to epithelial cells to prevent passive 

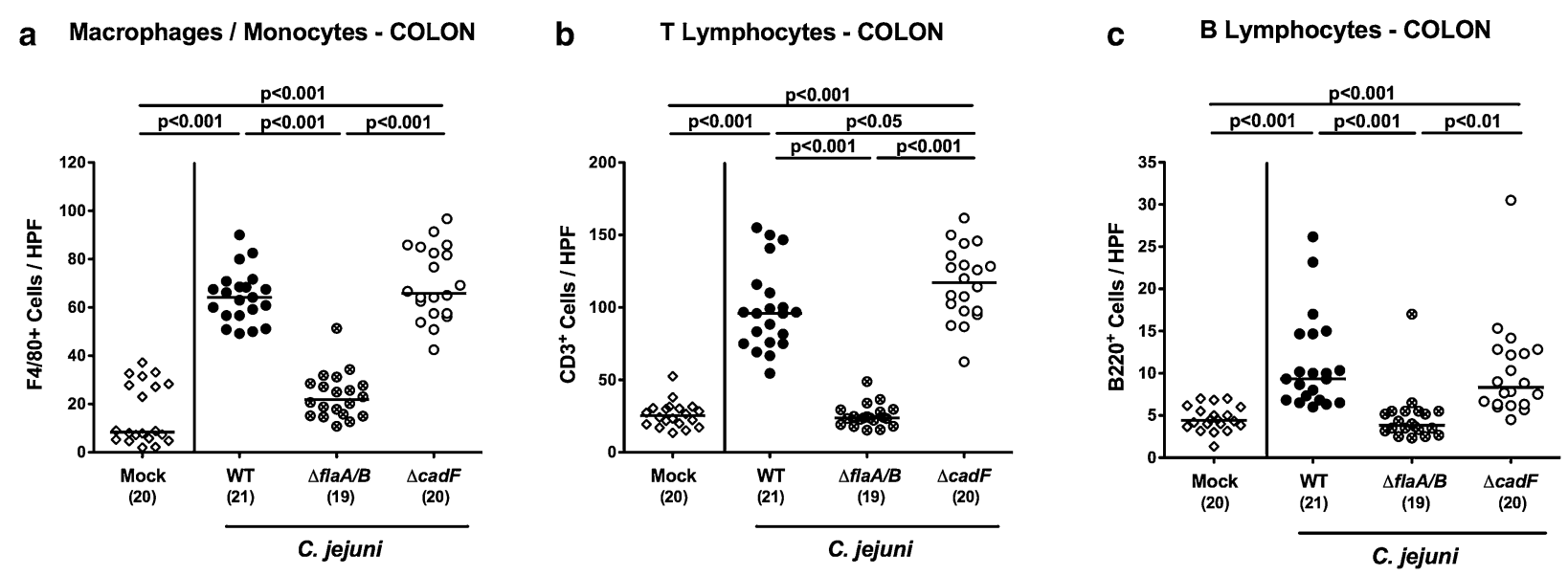

Fig. 5 Immune cell responses in the large intestine. The average numbers of immune cells were determined microscopically from six HPF ( $\times 400$ magnification) per infected or control animal using immunohistochemically stained colonic paraffin sections. Shown are data for a macrophages and monocytes (F4/80+), b T lymphocytes (CD3+), and c B lymphocytes (B220+)

transport to the colon where even the non-motile bacteria accumulate due to the low peristaltics.

It is well established that flagella-deficient C. jejuni mutants are unable to colonize the gastrointestinal tract of infant mice [38, 39], of young chicks [40] and of piglets [41]. However, a role of $\mathrm{CadF}$ in colonisation of the mammalian gut was mostly inferred from in vitro data, as it was shown previously that cadF-deficient $C$. jejuni mutants poorly adhered to cultured mammalian cells $[21,25,34]$, though CadF was reported to be essential for cecal colonization in chicken [26]. Our data provide strong evidence, however, that CadF is not essential for murine colonization, and only marginally affects the outcome of infection. Thus, one or more different adhesin(s) are obviously required for $C$. jejuni colonization and disease development in mice. Because this might reflect the situation in humans, it will be important to identify these factors in further screens.

The difference between mutants deficient in flagellins or $c a d F$ extended beyond colonization capacity, given that there were also noted differences in their ability to generate macroscopic disease signs and microscopic inflammatory responses in the large intestines. Interestingly, WT and cadF deficient bacteria were able to enhance pro-inflammatory mediator secretion in distinct compartments of the intestinal tract, which was not seen with the non-motile $C$. jejuni mutant lacking flagella. The murine model applied here also allowed to investigate the capacity to generate campylobacteriosislike symptoms in mice. Surprisingly, high numbers of $C$. jejuni present in the colon were not per se responsible for triggering disease, as could be demonstrated with the $\triangle f l a A / B$ mutant that colonized the colon effectively, but did not cause enteric disease. In a recent study applying a murine $C$. jejuni induced enteritis model, however, mice could not be infected by a flaA-deficient mutant strain and did therefore not display any signs of enteritis [42]. Notably, in this study the gut microbiota of corresponding mice was not completely eradicated and this leads to the assumption that the residual microbiota established after vancomycin treatment is responsible for the complete colonization defect of the flaA deficient mutant. This provides evidence that motility is required to allow C. jejuni to escape from commensal bacteria that produce harmful metabolites and thus create an unfavorable environment for the pathogen.

In our study, the presence of symptoms coincided with the ability to generate intestinal, extra-intestinal and systemic immune responses, which both WT bacteria and the $\Delta c a d F$ mutant were capable of. This observation further confirms the hypothesis of an immunopathological nature of campylobacteriosis. It has been hypothesized that bacterial invasion into host cells is regulated by proinflammatory mediators in the gut [43] and this idea is in line with our observations, since non-motile mutant strains, that are impaired in their capacity to invade concurrently exhibited far weaker immune responses.

A marked difference was further observed in the ability of $C$. jejuni to reach extra-intestinal sites. All three bacterial strains could be isolated from extra-intestinal organs, but the numbers of viable bacteria were much lower for the non-motile $\triangle f l a A / B$ mutant. As expected, it appeared that due to lack of flagella-dependent motility, fewer bacteria were able to reach liver, lungs, and spleen. Alternatively, it has been shown that the flagellum of $C$. jejuni can act as a type III secretion apparatus for the delivery 




c Nitric Oxide - COLON

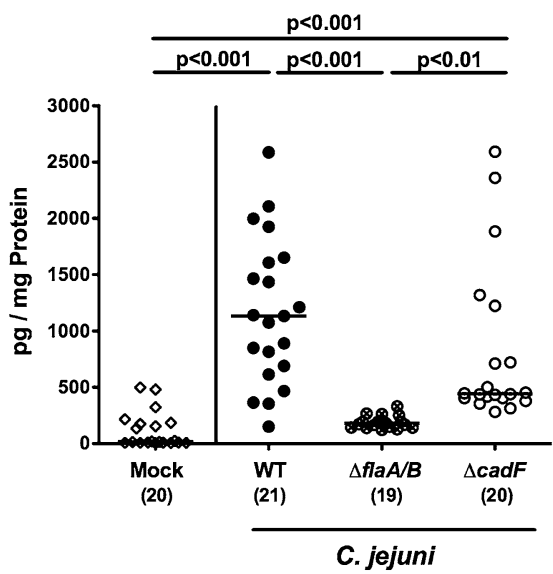

e

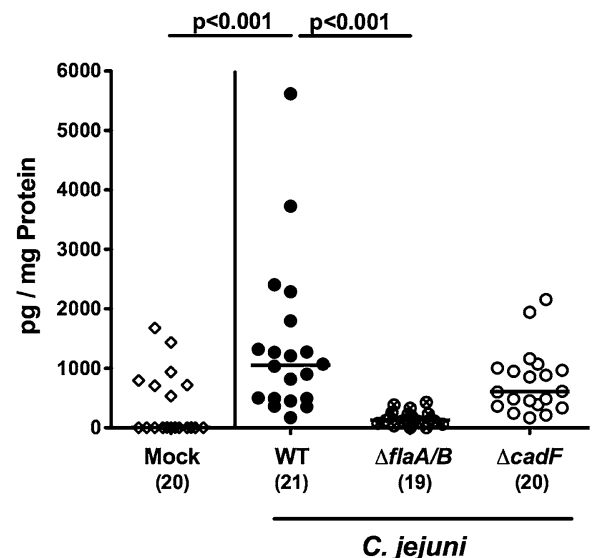

b

IL-6 - COLON

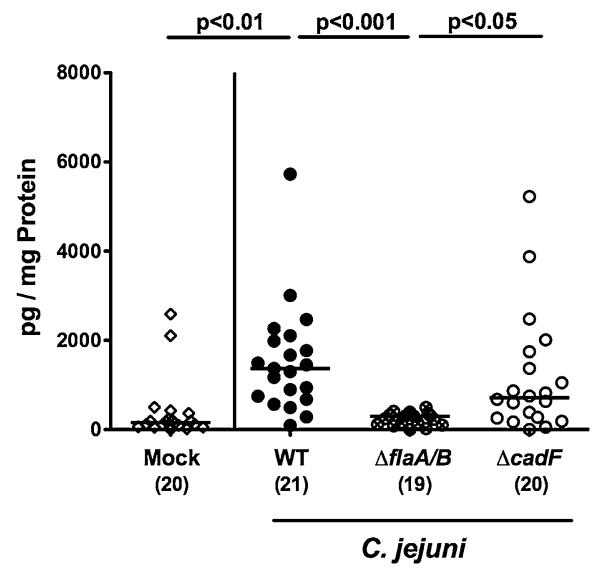

d

IFN- $\gamma=$ COLON

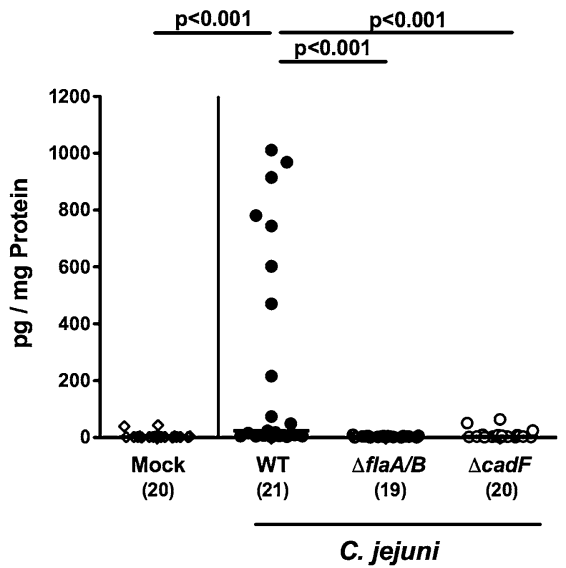

f

IFN- $\gamma=$ MLN



Fig. 6 Intestinal pro-inflammatory mediator responses. Levels were determined for colonic a TNF-a, b IL-6, c nitric oxide, and $\mathbf{d}$ IFN- $\gamma$ and for e nitric oxide and $\mathbf{f} \mid \mathrm{FN}-\gamma$ in supernatants of ex vivo biopsies derived from mesenteric lymph nodes (MLN) 
a T Lymphocytes - LIVER

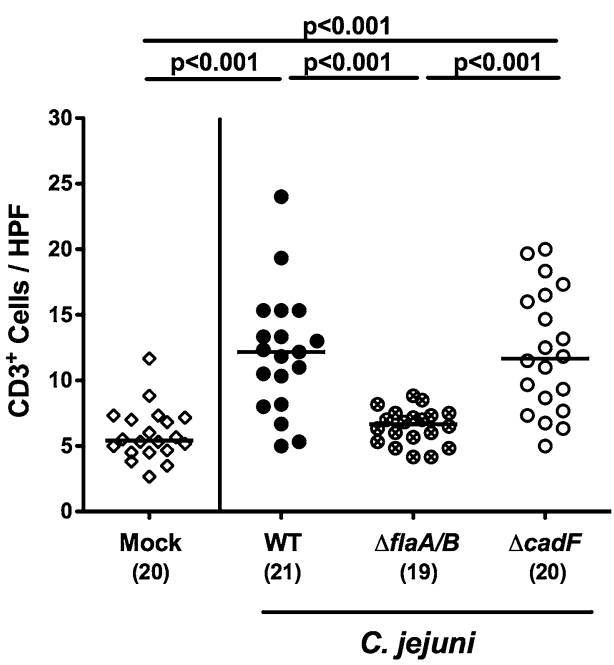

c

\section{TNF- $\alpha$ - LIVER}

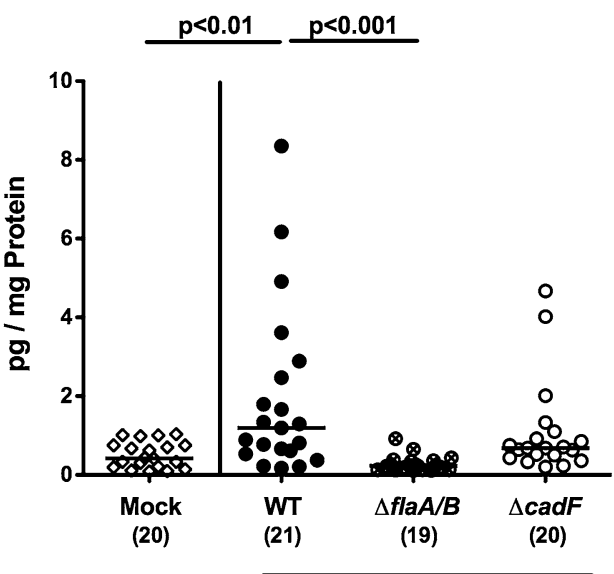

C. jejuni b T Lymphocytes - KIDNEY

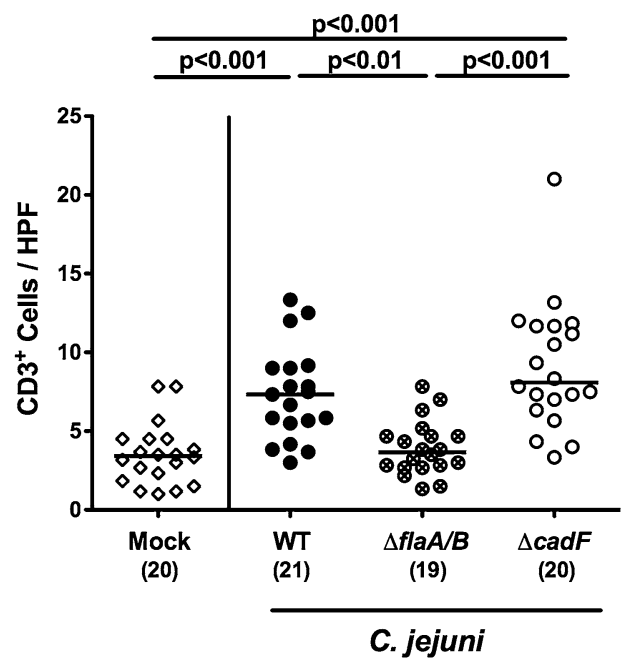

d

TNF- $\alpha$ - KIDNEY

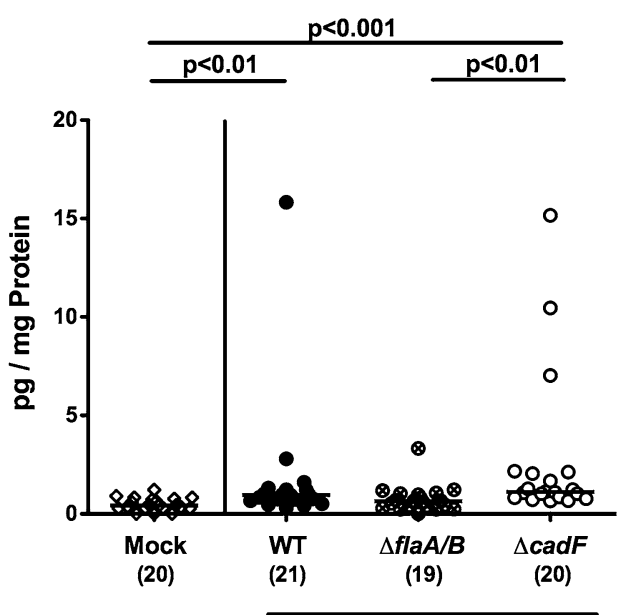

C. jejuni

Fig. 7 Pro-inflammatory immune responses in extra-intestinal tissues. Data are shown for T lymphocytes (CD3+) (a, b) and for TNF-a concentrations ( $\mathbf{c}, \mathbf{d})$ in liver $(\mathbf{a}, \mathbf{c})$ and kidneys $(\mathbf{b}, \mathbf{d})$ of infected and control animals

of bacterial factors such as the Cia or Fed proteins into the extracellular milieu or directly into host cells in vitro [18-20]. Thus, certain exported C. jejuni proteins may also trigger the above responses in mice. Thus, future studies should be designed to provide evidence for one or both of these options.

\section{Conclusion}

For the first time, our presented in vivo data provide evidence that $C$. jejuni FlaA/B, but not CadF are pivotally involved in inducing campylobacteriosis upon peroral infection of the vertebrate host. Future studies should unravel the underlying mechanisms of the host-pathogen interactions in more detail. Furthermore, the here applied clinical murine infection model of secondary abiotic IL- $10^{-/-}$mice has been proven suitable not only for detailed investigations of immunological aspects of 
a

TNF- $\alpha$ - SERUM



C. jejuni

c

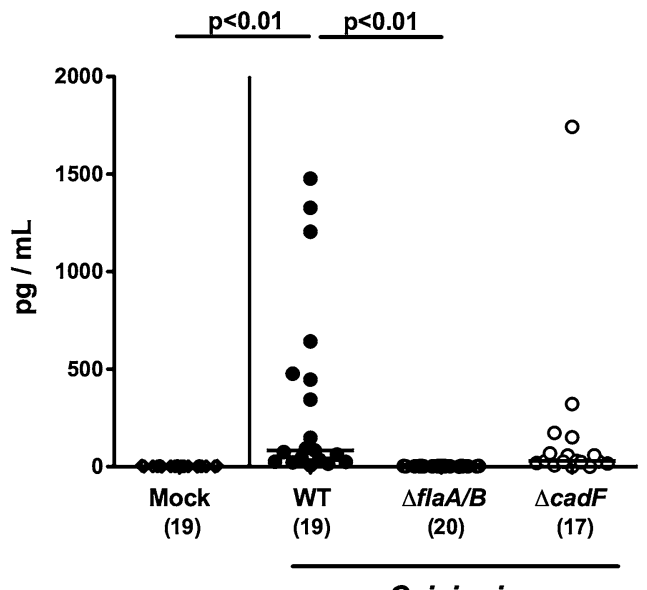

C. jejuni b

IL-6 - SERUM

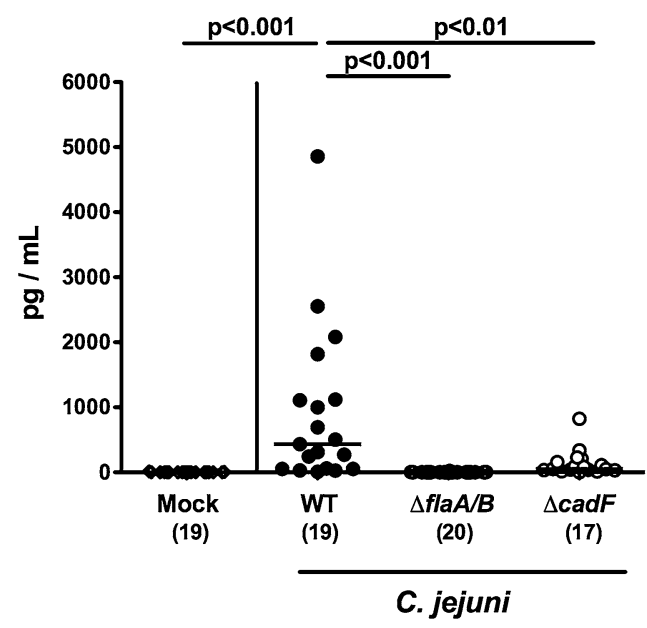

d

MCP-1 - SERUM
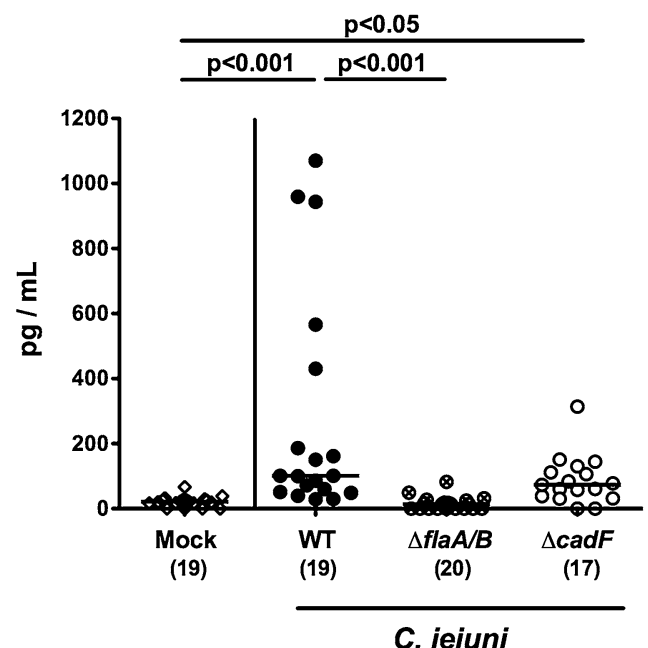

Fig. 8 Systemic pro-inflammatory mediator responses following C. jejuni infection. Data are shown for a TNF-a, b IL-6, c IFN-ץ, and $\mathbf{d} M C P-1$ in serum samples taken 6 days post-infection

campylobacteriosis, but also for differential analyses of the roles of distinct $C$. jejuni virulence factors in induction and progression of disease.

\section{Methods}

\section{Ethics approval}

All animal experiments were conducted in accordance with the European Guidelines for animal welfare (2010/63/EU) following approval of the protocol by the commission for animal experiments headed by the "Landesamt für Gesundheit und Soziales" (LaGeSo, Berlin, registration number G0247/16). Clinical conditions of mice were surveyed twice daily.

\section{Generation of secondary abiotic mice and C. jejuni} infection

IL-10 ${ }^{-/-}$mice of C57BL/6j background were reared and housed under specific pathogen free conditions. In order 


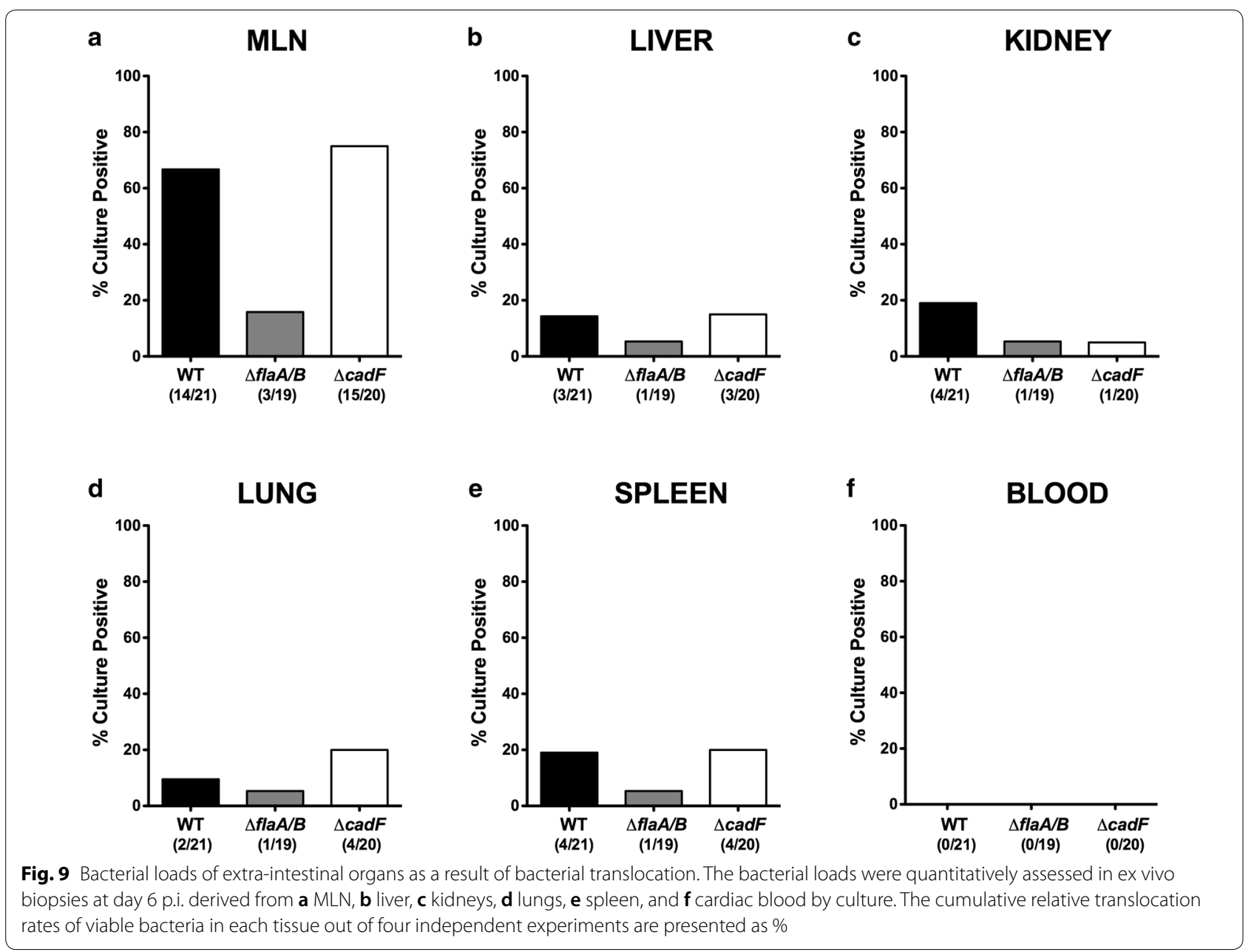

to counteract physiological colonization resistance and hence facilitate intestinal pathogenic colonization, secondary abiotic mice with a virtually depleted gut microbiota were generated upon broad-spectrum antibiotic treatment as reported earlier [31,33].

Sex-matched, 3 months old mice were perorally infected with either the $C$. jejuni parental strain 81-176 (WT), the isogenic $f l a A / B$ deletion mutant $(\triangle f l a A / B)$, or the $c a d F$ deletion mutant $(\triangle c a d F)$. An inoculum of $10^{9}$ CFU in $0.3 \mathrm{~mL}$ phosphate buffered saline (PBS; Gibco, life technologies, UK) was administered on two consecutive days (i.e., days 0 and 1) by oral gavage. Mock control animals received an equal volume PBS perorally. Mice were maintained in a sterile environment and had unlimited access to autoclaved food and drinking water and were handled under strict aseptic conditions to avoid contamination.

\section{Monitoring of clinical conditions}

The clinical conditions of the mice were surveyed prior and post respective $C$. jejuni infections on a daily basis by applying a standardized cumulative clinical score (maximum 12 points). These scores included the abundance of blood in feces as detected by the Guajac method using a Haemoccult, Beckman Coulter (PCD, Krefeld, Germany) (score 0: no blood; 2: microscopic detection of blood; 4: macroscopic blood visible), presence of diarrhea (score 0: formed feces; 2: pasty feces; 4: liquid feces), and by visual clinical and behavioral symptoms (score 0: normal; 2: ruffled fur and/or less locomotion; 4: isolation, severely compromised locomotion, pre-final aspect) as described earlier [29].

\section{Sampling procedures}

At day 6 post-infection (p.i.), the animals were sacrificed upon isoflurane inhalation (Abbott, Germany). Luminal gastrointestinal samples from stomach, duodenum, ileum and colon, and ex vivo biopsies from colon, 
ileum, mesenteric lymph nodes (MLN), liver, kidneys, lungs, and spleen were taken under sterile conditions. Intestinal samples were collected from each mouse in parallel for microbiological, immunohistopathological and immunological analyses. The absolute colonic lengths were measured with a ruler (in $\mathrm{cm}$ ).

\section{Immunohistochemistry}

In situ immunohistochemical analyses were performed in colonic ex vivo biopsies that had been immediately fixed in $5 \%$ formalin and embedded in paraffin as described earlier [44-46]. Paraffin sections $(5 \mu \mathrm{m})$ of ex vivo biopsies from colon, liver and kidneys were stained with primary antibodies directed against cleaved caspase 3 (Asp175, Cell Signaling, Beverly, MA, USA, 1:200) for detection of apoptotic epithelial cells; against Ki67 (TEC3, Dako, Denmark, 1:100) for detection of proliferating epithelial cells; against F4/80 (\# 14-4801, clone BM8, eBioscience, San Diego, CA, USA, 1:50) for detection of macrophages/monocytes; against CD3 (\#N1580, Dako, 1:10) for detection of T lymphocytes; and against B220 (No. 14-0452-81, eBioscience; 1:200) for detection of B lymphocytes. Secondary antibodies were used for detection as previously described [31, 47]. Positively stained cells were examined by light microscopy (magnification $100 \times$ and $400 \times$ ), and for each mouse the average number of respective positively stained cells was determined within at least six high power fields (HPF, $0.287 \mathrm{~mm}^{2}, 400 \times$ magnification) by an independent investigator using blinded samples.

\section{Bacterial colonization}

The number of viable C. jejuni bacteria was quantitatively assessed in feces over time p.i., in homogenates of ex vivo biopsies taken MLN, spleen, liver, kidneys and lungs, and in cardiac blood at day 6 p.i. by culture as described elsewhere [31, 47]. The detection limit of viable bacteria was $\approx 100 \mathrm{CFU}$ per $\mathrm{g}$.

\section{Pro-inflammatory mediator detection in supernatants of intestinal and extra-intestinal ex vivo biopsies}

Colonic ex vivo biopsies were cut longitudinally, washed in PBS, and strips of approximately $1 \mathrm{~cm}^{2}$ tissue as well as ex vivo biopsies derived from MLN (3 lymph nodes), liver (approximately $1 \mathrm{~cm}^{3}$ ), one kidney (cut longitudinally), and one lung were placed in 24-flat-bottom well culture plates (Nunc, Germany) containing $500 \mu \mathrm{L}$ serum-free RPMI 1640 medium (Gibco, life technologies, UK) supplemented with penicillin $(100 \mathrm{U} / \mathrm{mL})$ and streptomycin $(100 \mu \mathrm{g} / \mathrm{mL}$; PAA Laboratories, Germany). After $18 \mathrm{~h}$ at $37{ }^{\circ} \mathrm{C}$, culture supernatants were tested for tumor necrosis factor- (TNF-) $\alpha$, interleukin (IL)-6, interferon (IFN)- $\gamma$, and monocyte chemoattractant protein (MCP)-1 by the Mouse Inflammation Cytometric Bead Array (CBA; BD Biosciences, Germany) on a BD FACSCanto II flow cytometer (BD Biosciences). Nitric oxide was measured by the Griess reaction as reported previously [33]. Systemic pro-inflammatory mediator concentrations were assessed in serum samples.

\section{Statistical analysis}

Medians and levels of significance were determined by one-way ANOVA test followed by Tukey post-correction for multiple comparisons (GraphPad Prism v7, USA). Two-sided probability ( $\mathrm{p}$ ) values $\leq 0.05$ were considered significant. Experiments were reproduced three times and pooled data are shown.

\section{Additional file}

Additional file 1: Figure S1. Representative photomicrographs illustrating apoptotic and proliferating colonic epithelial as well as immune cells responses in large intestinal and extra-intestinal compertments in secondary abiotic $\mathrm{IL}-10^{-/-}$mice following peroral $\mathrm{flaA} B \mathrm{~B}$ or cadF gene deficient $C$. jejuni infection. Secondary abiotic $\mathrm{IL}-10^{-1-}$ mice were perorally challenged either with the C. jejuni 81-176 wildtype strain (WT), the isogenic $f l a A / B$ gene deletion mutant $(\triangle f l a A / B)$ or the isogenic cadF gene deletion mutant $(\triangle c a d F)$ by gavage on days 0 and 1. Mock mice served as negative controls. Photomicrographs reepresentative for four independent experiments illustrate (A) apoptotic colonic epithelial cells (Casp3+), (B) proliferating colonic epithelial cells, large intestinal (C) macrophages and monocytes (F4/80+), (D) T lymphocytes (CD3+), (E) B lymphocytes $(B 220+)$ and furthermore, (F) hepatic and (G) renal T lymphocytes (CD3+) in at least six high power fields (HPF) as quantitatively assessed in respective paraffin sections applying in situ immunohistochemistry at day 6 post-infection (100× magnification, scale bar $100 \mu \mathrm{m})$.

\section{Abbreviations}

cadF: Campylobacter adhesin to fibronectin; CBA: Cytometric Bead Array; CFU: colony-forming units; FlaA/B: flagella filament A/B; HPF: high power field; IFN: interferon; IL: interleukin; MAPK/ERK: mitogen-activated protein kinases/extracellular signal-regulated kinases; MCP: monocyte chemoattractant protein; MLN: mesenteric lymph nodes; n.s.: not significant; NF-kB: nuclear factor kappa-light-chain-enhancer of activated B cells; Nod: nucleotide-oligomerization-domain; PBS: phosphate-buffered saline; p.i.: post-infection; TLR: Toll-like receptor; TNF: tumor necrosis factor; WT: wildtype.

\section{Acknowledgements}

We thank Alexandra Bittroff-Leben, Ines Puschendorf, Ulrike Fiebiger, Gernot Reifenberger, and the staff of the animal research facility at Charité-University Medicine Berlin for excellent technical assistance and animal breeding. We acknowledge support from the German Research Foundation (DFG), German Federal Ministries of Education and Research (BMBF) and the Open Access

Publication Fund of Charité-Universitätsmedizin Berlin.

\section{Authors' contributions}

AMS: Designed and performed experiments, analyzed data, co-wrote paper. SM: Performed experiments, analyzed data. UE: Performed experiments. NT: Provided bacterial strains, co-edited paper. MB: Co-edited paper. SBa: Provided advice in experimental design, critically discussed results, co-edited paper. SBe: Provided advice in experimental design, critically discussed results, 
co-edited paper. MMH: Designed and performed experiments, analyzed data, wrote paper. All authors read and approved the final manuscript.

\section{Funding}

This work was supported by the German Science Foundation (DFG) to MB (Project BO4724/1-1), MMH and UE (HE3040/3-1), as well as from the German Federal Ministries of Education and Research (BMBF) in frame of the zoonoses research consortium PAC-Campylobacter to SBe, MMH, SM (IP7/01KI1725D) and to SBa (IP9/01KI1725E).

The funders had no role in study design, data collection and analysis, decision to publish or preparation of the manuscript.

\section{Availability of data and materials}

Please contact author for data requests.

\section{Ethics approval}

All animal experiments were conducted in accordance with the European Guidelines for animal welfare (2010/63/EU) following approval of the protocol by the commission for animal experiments headed by the "Landesamt für Gesundheit und Soziales" (LaGeSo, Berlin, Registration Number G0247/16). Clinical conditions of mice were surveyed twice daily.

\section{Consent of publication}

Not applicable.

\section{Competing interests}

The authors declare that they do not have competing interests.

\section{Author details}

${ }^{1}$ Department of Microbiology, Institute of Microbiology, Infectious Diseases and Immunology, Charité-University Medicine Berlin, corporate member of Freie Universität Berlin, Humboldt-Universität zu Berlin, and Berlin Institute of Health, CC5, Campus Benjamin Franklin, FEM, Garystr. 5, 14195 Berlin, Germany. ${ }^{2}$ Institute for Microbiology, Department of Biology, Friedrich Alexander University Erlangen/Nuremberg, Erlangen, Germany.

Received: 6 April 2019 Accepted: 10 May 2019

Published online: 17 May 2019

\section{References}

1. Kist M, Bereswill S. Campylobacter jejuni. Contrib Microbiol Immunol. 2001:8:150-65.

2. Young KT, Davis LM, Dirita VJ. Campylobacter jejuni: molecular biology and pathogenesis. Nat Rev Microbiol. 2007;5:665-79.

3. Ó Cróinín T, Backert S. Host epithelial cell invasion by Campylobacter jejuni: trigger or zipper mechanism? Front Cell Infect Microbiol. 2012;2:1-13.

4. Burnham PM, Hendrixson DR. Campylobacter jejuni: collective components promoting a successful enteric lifestyle. Nat Rev Microbiol. 2018;16:551-65.

5. Kaakoush NO, Castaño-Rodríguez N, Mitchell HM, Man SM. Global epidemiology of Campylobacter infection. Clin Microbiol Rev. 2015;28:687-720.

6. Wassenaar TM, Blaser MJ. Pathophysiology of Campylobacter jejuni infections of humans. Microbes Infect. 1999;1:1023-33.

7. Backert S, Tegtmeyer N, Crónin T, Boehm M, Heimesaat MM. Human campylobacteriosis. In: Klein G, editor. Campylobacter-feature, detection, and prevention of foodborne disease. London: Elsevier; 2017. p. $1-16$.

8. Nachamkin I. Chronic effects of Campylobacter infection. Microbes Infect. 2002:4:399-403.

9. de Zoete MR, Keestra AM, Roszczenko P, van Putten JPM. Activation of human and chicken toll-like receptors by Campylobacter spp. Infect Immun. 2010;78:1229-38.

10. Heimesaat MM, Grundmann U, Alutis ME, Fischer A, Bereswill S. Absence of nucleotide-oligomerization-domain-2 Is associated with less distinct disease in Campylobacter jejuni infected secondary abiotic IL-10 deficient mice. Front Cell Infect Microbiol. 2017;7:1-13.

11. Heimesaat MM, Grundmann U, Alutis ME, Fischer A, Bereswill S. Small intestinal pro-inflammatory immune responses following Campylobacter jejuni Infection of secondary abiotic IL-10-1- mice lacking nucleotideoligomerization-domain-2. Eur J Microbiol Immunol. 2017:7:138-45.

12. MacCallum A, Haddock G, Everest PH. Campylobacter jejuni activates mitogen-activated protein kinases in Caco-2 cell monolayers and in vitro infected primary human colonic tissue. Microbiology. 2005;151:2765-72.

13. Chen ML, Ge Z, Fox JG, Schauer DB. Disruption of tight junctions and induction of proinflammatory cytokine responses in colonic epithelial cells by Campylobacter jejuni. Infect Immun. 2006;74:6581-9.

14. Hu L, Bray MD, Osorio M, Kopecko DJ. Campylobacter jejuni induces maturation and cytokine production in human dendritic cells. Infect Immun. 2006;74:2697-705.

15. Lugert R, Groß U, Zautner AE. Campylobacter jejuni: cornponents for adherence to and invasion of eukaryotic cells. Berl Münch Tierärztl Wochenschr. 2015;128:10-7.

16. Lertsethtakarn P, Ottemann KM, Hendrixson DR. Motility and Chemotaxis in Campylobacter and Helicobacter. Annu Rev Microbiol. 2011;65:389-410.

17. Guerry P. Campylobacter flagella: not just for motility. Trends Microbiol. 2007:15:456-61.

18. Barrero-Tobon AM, Hendrixson DR. Identification and analysis of flagellar co-expressed determinants (Feds) of Campylobacter jejuni involved in colonization. Mol Microbiol. 2012;84:352-69.

19. Eucker TP, Konkel ME. The cooperative action of bacterial fibronectinbinding proteins and secreted proteins promote maximal Campylobacter jejuni invasion of host cells by stimulating membrane ruffling. Cell Microbiol. 2012;14:226-38.

20. Scanlan E, Yu L, Maskell D, Choudhary J, Grant A. A quantitative proteomic screen of the Campylobacter jejuni flagellar-dependent secretome. J Proteomics. 2017:152:181.

21. Monteville MR, Yoon JE, Konkel ME. Maximal adherence and invasion of INT 407 cells by Campylobacter jejuni requires the CadF outermembrane protein and microfilament reorganization. Microbiology. 2003;149:153-65

22. Krause-Gruszczynska M, Rohde M, Hartig R, Genth H, Schmidt G, Keo T, et al. Role of the small Rho GTPases Rac1 and Cdc42 in host cell invasion of Campylobacter jejuni. Cell Microbiol. 2007;9:2431-44.

23. Wassenaar TM, Bleumink-Pluym NMC, van der Zeijst A, Bernard AM. Inactivation of Campylobacter jejuni flagellin genes by homologous recombination demonstrates that flaA but not $f a B$ is required for invasion. EMBO J. 1991;10:2055-61.

24. Yao R, Burr DH, Doig P, Trust TJ, Niu H, Guerry P. Isolation of motile and non-motile insertional mutants of Campylobacter jejuni: the role of motility in adherence and invasion of eukaryotic cells. Mol Microbiol. 1994;14:883-93.

25. Krause-Gruszczynska M, van Alphen LB, Oyarzabal OA, Alter T, Haenel I, Schliephake A, et al. Expression patterns and role of the CadF protein in Campylobacter jejuni and Campylobacter coli. FEMS Microbiol Lett. 2007;274:9-16.

26. Ziprin RL, Young CR, Stanker LH, Hume ME, Konkel ME. The absence of cecal colonization of chicks by a mutant of Campylobacter jejuni Not expressing bacterial fibronectin-binding protein. Avian Dis. 1999;43:586-9.

27. Boehm M, Krause-Gruszczynska M, Rohde M, Tegtmeyer N, Takahashi S, Oyarzabal OA, Backert S. Major host factors involved in epithelial cell invasion of Campylobacter jejuni: role of fibronectin, integrin beta1, FAK, Tiam-1, and DOCK180 in activating Rho GTPase Rac1. Front Cell Infect Microbiol. 2011;1:1-17.

28. Haag L-M, Fischer A, Otto B, Plickert R, Kuehl AA, Goebel UB, et al. Campylobacter jejuni Induces Acute Enterocolitis in Gnotobiotic IL-10 ${ }^{-1-}$ Mice via Toll-Like-Receptor-2 and -4 Signaling. PLoS ONE. 2012;7:1-11.

29. Heimesaat MM, Alutis M, Grundmann U, Fischer A, Tegtmeyer N, Boehm $M$, et al. The role of serine protease $H$ trA in acute ulcerative enterocolitis and extra-intestinal immune responses during Campylobacter jejuni infection of gnotobiotic IL-10 deficient mice. Front Cell Infect Microbiol. 2014:4:1-14.

30. Heimesaat MM, Bereswill S. Murine infection models for the investigation of Campylobacter jejuni-host interactions and pathogenicity. Berl Münch Tierärztl Wochenschr. 2015;128:98-103.

31. Bereswill S, Fischer A, Plickert R, Haag L-M, Otto B, Kuehl AA, et al. Novel murine infection models provide deep insights into the "Ménage à Trois" of Campylobacter jejuni, microbiota and host innate immunity. PLOS ONE. 2011;6:1-13. 
32. Otto B, Haag L-M, Fischer A, Plickert R, Kuehl AA, Goebel UB, et al. Campylobacter jejuni induces extra-intestinal immune responses via Toll-likereceptor-4 signaling in conventional IL-10 deficient mice with chronic colitis. Eur J Microbiol Immunol. 2012;2:210-9.

33. Heimesaat MM, Bereswill S, Fischer A, Fuchs D, Struck D, Niebergall J, et al. Gram-negative bacteria aggravate murine small intestinal Th1-type immunopathology following oral infection with Toxoplasma gondii. J Immunol. 2006;177:8785-95.

34. Moser I, Schroeder W, Salnikow J. Campylobacter jejuni major outer membrane protein and a 59-kDa protein are involved in binding to fibronectin and INT 407 cell membranes. FEMS Microbiol Lett. 1997;157:233-8.

35. Boehm M, Hoy B, Rohde M, Tegtmeyer N, Bæk KT, Oyarzabal OA, et al. Rapid paracellular transmigration of Campylobacter jejuni across polarized epithelial cells without affecting TER: role of proteolytic-active HtrA cleaving E-cadherin but not fibronectin. Gut Pathog. 2012;4:1-12.

36. Backert S, Boehm M, Wessler S, Tegtmeyer N. Transmigration route of Campylobacter jejuni across polarized intestinal epithelial cells: paracellular, transcellular or both? Cell Comm Signal. 2013;11:1-15.

37. Al-Banna NA, Cyprian F, Albert MJ. Cytokine responses in campylobacteriosis: linking pathogenesis to immunity. Cytokine Growth Factor Rev. 2018;41:75-87.

38. Morooka T, Umeda A, Amako K. Motility as an intestinal colonization factor for Campylobacter jejuni. J Gen Microbiol. 1985;131:1973-80.

39. Newell DG, McBride H, Dolby JM. Investigations on the role of flagella in the colonization of infant mice with Campylobacter jejuni and attachment of Campylobacter jejuni to human epithelial cell lines. J Hyg. 1985;95:217-27.

40. Wassenaar TM, van der Zeijst A, Bernard AM, Ayling R, Newell DG. Colonization of chicks by motility mutants of Campylobacter jejuni demonstrates the importance of flagellin A expression. J Gen Microbiol. 1993;139:1171-5.

41. Babakhani FK, Bradley GA, Joens LA. Newborn piglet model for campylobacteriosis. Infect Immun. 1993;61:3466-75.

42. Stahl M, Ries J, Vermeulen J, Yang H, Sham HP, Crowley SM, et al. A nove mouse model of Campylobacter jejuni gastroenteritis reveals key proinflammatory and tissue protective roles for Toll-like receptor signaling during infection. PLoS Pathog. 2014;10:1-16.

43. Singh A, Mallick Al. Role of putative virulence traits of Campylobacter jejuni in regulating differential host immune responses. J Microbiol. 2019;57:298-309.

44. Alutis ME, Grundmann U, Fischer A, Hagen U, Kuehl AA, Goebel UB, et al. The role of gelatinases in Campylobacter Jejuni infection of gnotobiotic mice. Eur J Microbiol Immunol. 2015;5:256-67.

45. Alutis ME, Grundmann U, Hagen U, Fischer A, Kuehl AA, Goebel UB, et al. Matrix metalloproteinase-2 mediates intestinal immunopathogenesis in Campylobacter Jejuni-infected infant mice. Eur J Microbiol Immunol. 2015:5:188-98.

46. Heimesaat MM, Giladi E, Kühl AA, Bereswill S, Gozes I. The octapeptide NAP alleviates intestinal and extra-intestinal anti-inflammatory sequelae of acute experimental colitis. Peptides. 2018;101:1-9.

47. Heimesaat MM, Haag L-M, Fischer A, Otto B, Kuehl AA. Survey of extraintestinal immune responses in asymptomatic long-term Campylobacter jejuni-infected mice. Eur J Microbiol Immunol. 2013;3:174-82.

\section{Publisher's Note}

Springer Nature remains neutral with regard to jurisdictional claims in published maps and institutional affiliations.
Ready to submit your research? Choose BMC and benefit from:

- fast, convenient online submission

- thorough peer review by experienced researchers in your field

- rapid publication on acceptance

- support for research data, including large and complex data types

- gold Open Access which fosters wider collaboration and increased citations

- maximum visibility for your research: over $100 \mathrm{M}$ website views per year

At BMC, research is always in progress.

Learn more biomedcentral.com/submissions 